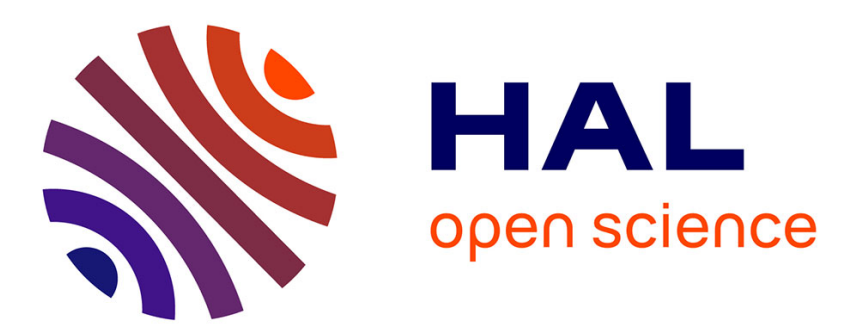

\title{
Gestualité humaine et langues des signes : entre continuum et variations
}

Dominique Boutet, Marie-Anne Sallandre, Ivani Fusellier-Souza

\section{To cite this version:}

Dominique Boutet, Marie-Anne Sallandre, Ivani Fusellier-Souza. Gestualité humaine et langues des signes: entre continuum et variations. Langage et Société, 2010, 131, pp.55-74. hal-00609029

\section{HAL Id: hal-00609029 \\ https://hal.science/hal-00609029}

Submitted on 1 Aug 2011

HAL is a multi-disciplinary open access archive for the deposit and dissemination of scientific research documents, whether they are published or not. The documents may come from teaching and research institutions in France or abroad, or from public or private research centers.
L'archive ouverte pluridisciplinaire HAL, est destinée au dépôt et à la diffusion de documents scientifiques de niveau recherche, publiés ou non, émanant des établissements d'enseignement et de recherche français ou étrangers, des laboratoires publics ou privés. 


\section{Gestualité humaine et langues des signes : entre continuum et variations \\ D. Boutet, I. Fusellier-Souza et M-A. Sallandre}

\section{Introduction}

Dans cet article, nous tenterons de revisiter la notion de continuum gestuel classiquement adoptée par les chercheurs en développant les fondements qui lient la gestuelle humaine signifiante aux langues des signes (LS), qu'elles soient en cours d'émergence ou déjà institutionnalisées. Nous analyserons quelques éléments de la variation et de la stabilisation de formes au sein du continuum et notamment les effets du passage de la bimodalité vers une seule modalité et, à ce titre ce qui distingue l'expression gestuelle de l'expression vocale. Notre réflexion s'appuie sur un modèle qui intègre la sémiologie du canal et vise l'ouverture de champs d'investigations concernant le lien entre la gestualité humaine et les LS :

1) les conséquences de l'utilisation du canal audio-phonatoire versus du canal visuo-gestuel et le rôle du visuel et du proprioceptif dans la dynamique structurante (et structurée) de ce dernier ;

2) l'expression gestuelle qui obéit à un tempo différent de celui des langues vocales, du fait de la compositionnalité de ses éléments ;

3) la manière dont les interrelations entre gestualité naturelle et LS peuvent rendre compte du phénomène de variation et d'invariants intra et inter-LS aux deux plans du développement de la LS chez l'individu (ontogenèse) et du développement historique de cette langue (phylogenèse).

Nous verrons successivement comment le continuum gestuel, structuré autour de la modalité vocale, linéarise des phénomènes pourtant multidimensionnels et comment une des instances - la pantomime - sert d'exutoire à des productions propres aux Langues des Signes (LS). Après avoir écarté la pantomime du continuum, nous exposerons en quoi ces productions dites de Grande Iconicité en LS se différencient de la pantomime et des signes standards. Enfin, nous verrons comment les LS émergentes se structurent à partir d'une exploitation de l'iconicisation de l'expérience et de la gestualité humaine, et brouillent l'agencement linéaire du continuum gestuel.

\section{Le continuum de Kendon : norme et modalités}

Adam Kendon (1988) définit le phénomène de gestualité symbolique comme celui d'un continuum qui va de la gesticulation idiosyncrasique en passant par la gestuelle coverbale puis, cheminant par la pantomime et les emblèmes, et finalement aux les langues signées (LS). Le continuum largement repris s'imposant ainsi comme une véritable norme scientifique (Kendon 1988, McNeill 1992, 2000, 2005, Singleton et al. 1995), en plus du cadre de référence qu'il installe, exerce une emprise sur la conception même du phénomène gestuel. Il le fait de deux manières. D'un côté, par l'écartement supposé de l'empan qu'il balaie - de la gesticulation jusqu'aux LS (voir schéma ci-dessous) - et, de 
l'autre, par la modalité visuo-gestuelle qu'il est censé intégrer. Suivant ce deuxième point, le continuum oriente la vision que l'on a de la gestualité à cause d'une approche linguistique non exempte des effets du canal audio-vocal. Aussi, interrogerons-nous ici ces deux dimensions : l'extensivité du continuum et les effets de la modalité visuo-gestuelle, réels d'une part, et, sousjacents à l'analyse, celle de la modalité audio-vocale, d'autre part. Les effets de canal avérés (visuogestuels) sont sans doute sous-estimées pour la gestualité ; aussi nous aborderons l'idée même de continuum par cette modalité. En ce qui concerne l'extensivité du continuum, nous montrerons qu'en son milieu ses instances - la pantomime et les emblèmes - constituent des catégories à resituer et à repenser. Redessiner les rapports entre les extrémités de ce continuum - gesticulation d'un côté et LS de l'autre - revient ici à replier la conception linéaire et plane de la continuité en une sorte de ruban de Möbius. Voyons en premier lieu, de quelles continuités il s'agit.

\subsection{Les quatre continua ou les variations autour d'une modalité}

A propos du continuum présenté dans son article princeps, Kendon appuie la démarche de McNeill dans la multiplication du nombre d'axes de continuité (Kendon 2004, p.106). Quatre continua caractérisent les instances gestuelles du continuum tel que cela est présenté ci-dessous.

Continuum 1 : relations à la parole Présence obligatoire..

.Absence obligatoire

Continuum 2 : relations aux propriétés linguistiques

Absence.

Présence

\section{Gesticulation >Gestuelle $>$ Pantomime $>$ Emblème $>$ Langue des Signes}

Continuum 3 : relations à la convention

Non conventionnelle...

Plein ement conventionnelle

Continuum 4 : relations à la sémiose

Globale et synthétique.

Segmentée et analytique

Tableau 1 : Le continuum de Kendon accompagné des 4 continua et de leurs caractéristiques

Le premier continuum voit décroître la présence de la parole de gauche à droite. Ainsi, dès la pantomime, on assiste à une absence de parole qui se poursuit évidemment jusqu'aux LS. Pour le deuxième continuum, celui des propriétés linguistiques, l'absence de contraintes phonologiques, de combinaisons syntaxiques de la gesticulation et dans une moindre mesure pour la gestuelle, font place à des contraintes de bonnes formes pour les emblèmes, puis, pour les LS, à une présence de l'ensemble des propriétés linguistiques. Par exemple, pour un geste relevant de la gesticulation et qui accompagne la parole, comme celui d'une saisie (poing fermé), la main à hauteur de visage et dont le mouvement du bras et de l'avant-bras forme un arc de cercle et amène la main refermée vers 
l'extérieur et l'arrière ${ }^{1}$, l'analyse selon ce deuxième continuum révèle une réalisation non phonologique, non morphémique et sans potentiel de combinaison syntaxique. Pour un emblème, McNeill voit une contrainte de bonne forme (le geste pour « $\mathrm{OK} »$, rond entre le pouce et l'index, ne peut se faire avec un mouvement oscillant de gauche à droite), sans la présence de combinaison syntaxique. En continuant, pour la LSF, un signe comme [ARBRE] pour lequel le coude repose sur le dos de l'autre main placée sur le côté tandis que l'avant-bras figurant le tronc se dresse vers le haut et, dans le prolongement, la main ouverte et les doigts écartés évoquent la ramure ; ce signe rentre dans un système de type phonologique, il peut se combiner avec un autre signe. D'une absence totale de propriétés linguistiques à gauche du continuum, on passe progressivement vers plus d'éléments linguistiques pour aboutir à une langue constituée. Le troisième axe, celui de la convention, voit celle-ci augmenter à mesure que les gestes se rapprochent d'une LS. Le quatrième continuum, sémiotique, voit d'un côté la gesticulation qualifiée de globale et synthétique, tandis qu'à l'autre extrémité, les signes des LS sont segmentés et analytiques ; voyons cela plus avant.

La gestuelle coverbale est qualifiée de globale par McNeill parce que la signification des composants d'un geste (type de mouvement, direction, configuration de la main, par exemple dans le geste de saisie évoqué précédemment d'arrachage d'un arbre) est déterminée par la signification de l'ensemble. Ainsi, la séquencialité avérée des signes dans les LS, redevables d'un fait linguistique pour McNeill, ferait-elle le pendant à la séquencialité des langues vocales (LV). Pourtant, il nous semble que cette séquencialité dans les LV pourrait provenir d'un effet de canal (linéarité vocale) et serait par là moins linguistique que modale. De ce fait, elle ne devrait pas pouvoir s'étendre à des LS dont la modalité visuo-gestuelle exploite la simultanéité. Néanmoins, une séquencialité similaire aux LV existe bel et bien dans les LS, celle-ci relève vraisemblablement d'une pulsation de production approchante entre LS et LV, mais il y a loin entre la pulsation d'une production et la structuration de son contenu de bas niveau. Ainsi, d'une part, la multidimensionnalité gestuelle offre une possibilité de composition simultanée (la main agrippe l'équivalent d'un arbre et le mouvement suivant le met à bas en le tordant d'une manière particulière et dans une direction donnée) tandis que la linéarité en tant qu'effet de canal de la vocalité égrène ces unités successivement, d'autre part. Il serait curieux que l'utilisation temporelle de productions sémantisées soit la même pour la gestualité et pour la vocalité. Les gestes coverbaux constitués de compositions d'éléments rassemblent en un tournemain ce que le verbal/vocal co-occurrent dispose consécutivement. Ainsi, contrairement à ce qu'en dit McNeill, la gestuelle coverbale n'est pas globale, elle concentre des éléments là où la parole est obligée de les égrener. On peut affirmer également que la gestuelle n'est pas synthétique, et que les LS ne sont pas plus analytique (voir continuum 4 dans le schéma 1) : les deux extrémités gestuelles partagent une compositionnalité des

1 Il s'agit d'un geste figurant l'arrachage d'un arbre (McNeill, 2005, p.6) 
éléments en présence dans le geste ; il ne peut en être autrement. En effet, un geste de la main se situe dans un emplacement particulier, à tout moment la paume a une orientation donnée, la main entretient une conformation avec les doigts que les études de LS appellent configuration, enfin un mouvement est nécessaire pour mettre en place un geste (Stokoe 1960 et 1976, Cuxac 2000). Les quatre paramètres manuels qui sont porteurs de sens dans la LSF (Cuxac 2000 et 2003) et les LS émergentes (Fusellier-Souza, 2004), existent aussi de manière obligatoire pour la gestuelle (Boutet 2001 et 2008) ; même si pour cette dernière, ces paramètres sont a priori moins chargés de sens que
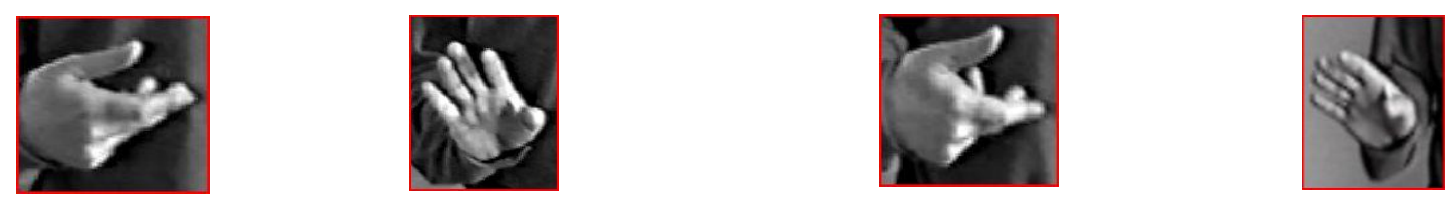

pour les LS. Dès lors, au moins quatre morphèmes (correspondants aux paramètres) pour chaque main, pour ne retenir que la composante manuelle d'un signe, sont exprimables simultanément en LS. Le tempo similaire entre LV et LS produit un mot ou un signe dans des durées semblables, mais le nombre potentiel de morphèmes par unité de temps est bien supérieur pour les LS, en particulier pour les structures de grande iconicité (SGI) présentées plus loin.

Le 4e continuum - de la sémiose - que l'on vient de voir, semble disparaitre en se rabattant entièrement sur le 1er, celui de la présence/absence de la parole. Ceci n'empêche pas la dépendance de la gestuelle à la parole co-occurrente, tant dans les formes parfois seulement esquissées que dans l'assujettissement du sens des gestes au discours prononcé. Cependant cette vision linguistique trop emprunte de phonocentrisme diffuse y compris sur l'analyse de la gestuelle. A ce titre, la place des emblèmes et de la pantomime dans le continuum relève certes d'une vocalité (par son absence), mais la présence de la pantomime est proche d'une convention. On va le voir.

\subsection{Les emblèmes et leurs esquisses}

Le fait que la catégorie des emblèmes occupe cette place en entre-deux dans le continuum pourrait revenir justement à une convention : celle d'une primauté de la parole dans la manière dont on appréhende la constitution même du sens dans la gestuelle. Ainsi, plutôt que de considérer en creux que les emblèmes sont des gestes plus aboutis par leur forme et leur sens que la gestuelle coverbale, on peut renverser le problème. Et considérer alors que la gestuelle coverbale met en œuvre des esquisses d'emblèmes, silhouettes non abouties ou dégradées parce que le discours en présence sous sa forme vocale suffit à faire sens. L'approche défendue ici revient à appliquer une primauté du sens à la gestualité tout en se gardant d'une collusion entre les effets de la modalité du phénomène dominant (linéarité et arbitrarité des LV) avec la nature linguistique du phénomène lui-même et surtout de celui qui l'accompagne (la gestuelle). Pour étayer cela, un test de reconnaissance portant sur des réalisations différentes par les segments mis en mouvement (doigts seulement [d], main et 
doigts [m], main et avant-bras [am] et avant-bras seul [a]) de quatre unités gestuelles a donné des résultats montrant que l'étiquetage des gestes ne se fait pas au hasard. Selon le protocole de cette méthode des juges, 4 réalisations segmentales par unité gestuelle (voir tableau 1) étaient présentées dans un ordre aléatoire et sans contexte verbal à 51 personnes. Sur un exemplier composé de 8 étiquettes (4 dans la cible, 4 en dehors), les sujets devaient assigner chaque réalisation gestuelle à une étiquette. Alors même que les réalisations gestuelles présentées ont été effectuées sans chercher à s'approcher d'un geste canonique, les réponses mesurées à l'aune de la loi binomiale (qui calcule la probabilité de l'apparition du hasard) sont probantes. Rappelons qu'on considère qu'une probabilité que le hasard intervienne dans le choix d'une étiquette inférieure à $5 \%(5 * 10-2)$ correspond à un seuil de signification.

\begin{tabular}{|c|c|c|c|c|c|c|c|c|c|c|c|}
\hline rejeter & $\mathrm{d}$ & $2,79 * 10^{-7}$ & refuser & $\mathrm{d}$ & $3,17 * 10^{-3}$ & passer & $\mathrm{d}$ & $4,77 * 10^{-2}$ & accepter & $\mathrm{d}$ & $2,12 * 10^{-2}$ \\
\hline & $\mathrm{m}$ & $2,79 * 10^{-7}$ & & $\mathrm{~m}$ & $3,73 * 10^{-1}$ & & $\mathrm{~m}$ & $5,83 * 10^{-14}$ & & $\mathrm{~m}$ & $9,49 * 10^{-5}$ \\
\hline & $\begin{array}{l}\mathrm{a} \\
\mathrm{m}\end{array}$ & $5,35^{*} 10^{-8}$ & & $\mathrm{am}$ & $3,32 * 10^{-4}$ & & am & $1,35 * 10^{-6}$ & & am & $8,59 * 10^{-3}$ \\
\hline & $\mathrm{a}$ & $6,03 * 10^{-6}$ & & $\mathrm{a}$ & $3,02 * 10^{-1}$ & & $\mathrm{a}$ & $1,35 * 10^{-6}$ & & $\mathrm{a}$ & $1,07 * 10^{-3}$ \\
\hline
\end{tabular}

Tableau 2 : Probabilités d'intervention du hasard dans le choix de l'étiquette assignée à chaque réalisation gestuelle

On voit que seules les étiquettes de 2 réalisations ont été choisies au hasard (grisé dans le tableau 2) Toutes les autres répondent largement à un choix d'étiquettes hautement significatif. Ces gestes non répertoriés comme des emblèmes sont pourtant reconnus et identifiés, ce malgré des réalisations différentes. Ces premiers résultats montrent d'abord que ces unités gestuelles sont organisées sur le substrat physiologique articulaire, preuve en est que les variations de mouvement de chaque unité sont sans impact sur la reconnaissance. Par ailleurs, une proximité entre le sens et la forme existent, interrogeant le caractère conventionnel de ces gestes : les antonymes [rejeter] vs [passer] ou bien [refuser] vs [accepter] présentent des pôles opposés des degrés de liberté en mouvement, renvoyant ainsi la convention dans une boucle oppositive sans issue. En outre, la géométrie de chaque segment (doigts, main, avant-bras et bras) différente par nature ne forme pas les mêmes réalisations. Malgré cela, la modalité visuelle pourtant capable de faire la différence entre deux réalisations manuelles (m) de deux unités gestuelles très proches par leur schémas d'action ([rejeter] et [refuser]), est par contre insensible à des variations de forme des réalisations gestuelles d'une même unité sur plusieurs segments (doigts, main ou avant-bras) puisque l'unité est bien reconnue (pour [rejeter] par exemple). Ainsi une modalité physiologique intervient dans la structuration des gestes et déborde même sur leur reconnaissance. Contrairement à ce qui a pu être dit (McNeill 2000) à propos d'une non composition des gestes coverbaux, il semble qu'une forme de concaténation soit possible. Le geste étiqueté [rejeter], déplaçable dans l'espace, lorsqu'il est effectué au dessus de l'épaule pourrait 
être paraphrasé par [négliger] ${ }^{2}$ prenant dans cette position particulière le sens attachée à la fin d'une réalisation mano-brachiale d'une autre unité étiquetée [s'en fiche].

Des gestes coverbaux, ou du moins répertoriés comme tels, et qui sont de fait des emblèmes montrent la porosité de ces notions. Une bonne reconnaissance du sens de ces gestes révèle de plus que le $3 \mathrm{e}$ continuum, celui de la convention est inopérant (opposition sémantique doublée d'une opposition formelle menant à une aporie de la conventionnalité). Une vision linéaire et monomodale de la gestuelle est remise en cause, la structuration de cette dernière, en sus de la vision comme modalité, peut être corporelle à travers la physiologie articulaire. Enfin, une partie de l'opacité de la signification des gestes (gesticulation) et de leur caractère idiosyncrasique pourrait provenir de la seule saisie visuelle de la forme gestuelle au détriment d'une reconnaissance proprioceptive. La vision saisit une forme sans sa structure physiologico-sémantique quand c'est le cas, et donc sans la signification qui y est alors attachée.

\subsection{Tentative définitoire de la pantomime}

Le second entre-deux du continuum de Kendon - la pantomime - à la fois exutoire, référence et enjeux de significations du corps depuis l'Antiquité (Graf 1991, Schmitt, 1990) n'échappe pas tout au long de l'histoire européenne à sa relégation voire à son éviction au nom de la valeur/vertu sociale et religieuse de la modestie. Augustin, au nom d'une des quatre vertus cardinales rejette la pantomime avec le théâtre qu'il abhorre (De Civ.Dei. I, XXXII ; Conf. 3, II, 4) tout en faisant rentrer les gestes des histrions ou des sourds dans une catégorie sémiologique (De Doc.Chr., 2, 1, 2 à 4) : attitude paradoxale de rejet et de référence. Pour plusieurs siècles la pantomime cristallise une bonne part de la charge que la société religieuse redoute, celle d'une gesticulation et d'une mimique gênante au regard du principe du fors intérieur (foris l'extérieur et donc le corps) comme reflet de l'état de l'âme (intus, intérieur). La pantomime au XVIIIe siècle constitue un enjeu pour l'origine du langage chez Condillac (1746) et un enjeu de compréhension de la pensée pour Diderot (1751), une référence constante et définitoire à l'Antiquité (du dictionnaire d'Estienne en 1552 jusqu'au Littré 1863 ; abbé DuBos 1993 [1719] ; de Jorio, 1832 ; Mallery, 1881), un exutoire religieux (voir Schmitt, 1991), social (Quint. Inst. 11.3-102), artistique à travers la mimique (Della Porta, 1586 ; Lebrun, 1806, [1668] pour n'en citer que deux). Le XIXe et le XXe siècle ne dérogent pas à ces trois caractéristiques : la pantomime est ballotée dans un jeu de références, entre des enjeux linguistiques et théâtraux et dans un exutoire où, au regard d'une tradition formelle de la linguistique, sont déversées les nombreuses saynettes faites par des sourds (de fait des Structures de Grande Iconicité, voir infra), parce que considérées comme non-langagières, dans ce fourre-tout qui relèverait plus du théâtre que du langage. La pantomime et ses possibilités sont fantasmées : âge

2 Le geste [REJETER] est bien identifiable avec, pour cet emplacement, cette pointe de désintérêt porté à la chose. 
pré-babelien, âge d'or antique, universalisme de l'expression des passions. Il s'agit donc avant tout d'en définir les contours moins à partir d'écrits historiques dont on a vu la relativité qu'en se référant aux productions des grands mimes contemporains.

Prenons le plus emblématique, le personnage créé par Marcel Marceau appelé Bip. Il fait apparaître ex nihilo des formes invisibles auxquelles il se heurte (vitre, vent), des matières par leurs résistances (cordes, poids) ou bien des actions par leur contraire (montée d'un escalier par un jeu de pied qui écrase chaque marche et une main qui descend par saccades, course qui se révèle au ralenti, illusion de la marche malgré une absence de déplacement, voire par un déplacement général vers l'arrière), des états par des accidents (Bip désespéré qui ne peut plus arracher le masque du rire). Une inversion générale laisse voir la réalité par son contraire : présence par l'absence, vitesse par le ralenti, désespoir par le rire. Dans cet exercice, le corps entièrement tourné vers l'émergence et la permanence de cette chose (objet ou situation) ne peut se morceler. La pantomime fonctionne par simulacre, cette apparence sensible qui se donne pour la réalité. Ainsi pour que la pantomime existe, le corps pris globalement (non morcellement) adhère au monde dans le sens qu'il le révèle par effets consécutifs du modelé que l'objet ou la situation opère sur lui ; cette adhérence d'ailleurs confine le corps à lui-même. En plus de ces deux caractéristiques importantes et liées de la pantomime - l'adhérence et la globalité - une troisième en rapport avec le 4e continuum concerne sa sémiose. On peut qualifier la pantomime de style (ainsi définie par Marceau) comme une pantomime de l'effet dans la mesure où ce qui est présenté d'un objet ou d'une situation passe indéfectiblement par l'effet physique exercé par eux sur le corps. On se situe d'emblée dans l'action ou dans l'acte et non dans le dire. Ce qui est présenté correspond à ce qui se passerait dans la réalité selon une boucle de rétroaction mêlant caractères physiques et physiologiques. Il n'y a pas production symbolique mais mimesis de la réalité. A cet égard, la pantomime sort de la continuité sémiologique des phénomènes de gestualité symbolique. Prise une fois de plus comme exutoire, base de références non définies et enjeu, elle sert au sein du continuum de déversoir au trop plein que sélectionne le cadre linguistique des langues vocales dans les productions dites « pantomimiques » des langues signées.

Une part importante des productions en Langue des Signes est donc renvoyée vers la pantomime par une tradition linguistique anglo-saxone. Ces signes non standards appelés Structures de Grande Iconicité (SGI) dans l'approche initiée par Christian Cuxac $(1996,2000)$ répondent néanmoins à une autre structuration que celle des productions pantomimiques. Ces structures sont issues d'un processus sémiologique et communicatif complexe qui exploite l'iconicisation de l'expérience à partir de la ressemblance des formes gestuelles, des référents réels et des percepts visuels. Ce que nous allons voir dans la prochaine section. 


\section{Les structures de grande iconicité des langues des signes : lieu d'un continuum unique}

de la gestualité humaine signifiante

Les structures de grande iconicité (SGI) s'inscrivent dans le cadre de la visée illustrative de donner à voir, d'après l'hypothèse de C. Cuxac (voir l'introduction générale et l'article de l'auteur et Pizzuto, ce numéro). Ces unités gestuelles non standardisées sont présentes dans de nombreuses LS étudiées à ce jour et sont à peu près identiques d'une LS à l'autre ${ }^{3}$, car elles obéissent à des règles de construction communes. Ce sont ces règles que nous allons présenter ici.

\subsection{Bref inventaire des structures de grande iconicité}

Le bref inventaire dressé ici permettra, d'une part, d'entrevoir la créativité linguistique des SGI, d'autre part, de voir comment elles participent à la redéfinition du continuum de Kendon en offrant la possibilité d'inclure simultanément dans le discours des éléments hétérogènes : structures de transferts, signes standard et gestualité humaine.

Voyons tout d'abord en quoi consistent les trois principaux transferts mis en évidence par Cuxac (1996). Les transferts de forme et de taille (TTF) rendent compte de lieux, d'objets, ou de personnages en décrivant les contours de leur forme et de leur taille. Les marques non manuelles qualifient et référentialisent ces formes : la mimique faciale et le mouvement indiquent si la forme signée par les mains est petite, plate ou ronde par exemple, tandis que le regard, porté sur eux, la référentialise et permet au locuteur de «dire en montrant». Les transferts situationnels (TS) expriment cette fois le déplacement d'un actant du procès (effectué par la main dominante) par rapport à un locatif stable (effectué par la main dominée). Le regard remplit le même rôle que pour les autres transferts, il précède puis suit le mouvement. Enfin, dans les transferts personnels (TP), il s'agit, pour le locuteur, en s'effaçant de la situation d'énonciation, d'être et de montrer un autre en train d'accomplir une action. Il s'agit d'une prise de rôle complète, avec actant, procès et modalité. Le locuteur "devient" l'entité dont il parle, il y a incorporation. Notons que la pantomime ne saurait incorporer sans artifice autre chose qu'un homme, à cause de ce principe d'adhérence au monde. Pour les SGI, au contraire, à l'instar de ce qui est présenté dans le tableau 3, ci-dessous, le corps n'adhère pas au monde mais figure par exemple un cheval ${ }^{4}$, y compris par autre chose que ce que le corps possède pourtant (principe inverse de l'adhérence) : les doigts pour les oreilles du cheval en dépit des oreilles du locuteur, la crinière par la main malgré la présence des cheveux, l'index et le majeur figurant le saut des jambes antérieures alors qu'il aurait été si simple pour le locuteur de faire un saut.

3 A titre indicatif, les SGI représentent en LSF autour de $65 \%$ du total des unités du discours dans le genre narratif et $25 \%$ du total des unités dans le genre explicatif (Sallandre, 2003).

4 En transfert personnel, le locuteur a la possibilité d'incarner tout type d'entités, animées ou non animées. 
L'analyse d'un extrait de corpus vidéo au sein d'une séquence narrative permet d'illustrer ces transferts et de montrer les variations possibles pour exprimer un cheval, en fonction de la visée choisie par le locuteur (illustrative ou non). Ce locuteur est droitier : sa main dominante est donc sa main droite, sa main dominée, la gauche. Le tableau 3 montre un exemple de quatre unités courantes en LSF : l'image (1) présente le signe standard [CHEVAL], signe constitué à partir de la reprise d'une partie saillante de l'entité (ici, les oreilles). Le locuteur regarde son interlocuteur, face à la caméra, ce qui est un indice fort d'une visée non illustrative et de l'emploi d'un signe standard. L'image (2) montre le locuteur décrivant la forme de la crinière avec sa main dominante, en TTF. L'image (3) présente le cheval en train de sauter par-dessus la barrière, en TS, avec le découpage suivant: la main dominante figure le cheval tandis que la main dominée figure la barrière et son regard suit l'action de la main dominante. Enfin, l'image (4) présente le locuteur dans le rôle du cheval en TP ; son regard est alors celui du personnage transféré.

\begin{tabular}{|c|c|c|c|}
\hline (1) $00{ }^{\prime} 09$ & (2) 00 ' 11 & (3) 00 '31 & (4) $00 ' 14$ \\
\hline Signe standard & Transfert de taille et forme & Transfert situationnel & Transfert personnel \\
\hline «CHEVAL » & $\begin{array}{l}\text { «forme de la crinière du } \\
\text { cheval » (main droite) }\end{array}$ & $\begin{array}{l}\text { "le cheval (main droite) saute } \\
\text { par-dessus la barrière (main } \\
\text { gauche) » }\end{array}$ & $\begin{array}{l}\text { "le cheval galope avec } \\
\text { bonheur » }\end{array}$ \\
\hline
\end{tabular}

Tableau 3 : Exemple de quatre unités signées dans le récit du Cheval

Par ailleurs, ces trois transferts élémentaires peuvent se combiner, soit entre eux, soit avec un signe standard ou un pointage. Il s'agit alors pour le locuteur d'utiliser ses différents paramètres corporels (main dominante, main dominée, buste, regard, mimique faciale, labialisation) d'une manière discrète du point de vue de la morphologie et de la sémantique.

Si on considère toutes les combinaisons possibles, on aboutit à 24 catégories $^{5}$ (Sallandre, 2003).

Voyons ici seulement quelques combinaisons possibles de SGI. Commençons par le double transfert, qui est l'association simultanée d'un transfert situationnel et d'un transfert personnel. Notre corpus donne l'exemple d'un locuteur capable d'incarner simultanément un cheval, avec son buste, son regard et sa mimique faciale (en transfert personnel), tandis que sa main dominante

5 Ce nombre peut être plus élevé selon la manière dont on considère la combinatoire des paramètres. 
figure un oiseau qui l'observe, et sa main dominée figure une barrière que le cheval s'apprête à franchir (en transfert situationnel). Cet exemple montre que produire deux actants différents et un locatif simultanément est possible en LS, grâce à la quadridimensionnalité du canal et à un morcellement corporel important, contrairement à ce qui serait produit par un pantomime. On voit qu'à la globalité pantomimique, répond ici à l'opposé un morcellement du corps pour les SGI, tel qu'on peut le qualifier de démembrement symbolique maintenu qui interdit au locuteur de retrouver sa globalité corporelle et donc, à la différence de la pantomime, qui lui interdit d'exprimer le monde par les seuls effets qu'il exerce sur soi.

Ensuite, voyons brièvement le stéréotype de transfert personnel qui est la reprise d'une attitude culturellement marquée, d'un geste coverbal stéréotypé, dans un discours en LS, pour suggérer l'état mental ou physique du personnage, non pour le décrire ou le simuler. Dans un exemple où le locuteur signe «se frotter les mains », l'intention du locuteur est d'indiquer la réflexion et la perplexité de son personnage transféré.

Enfin, terminons cet inventaire par un dernier sous-ensemble de transferts de personne, celui qui associe transfert personnel et discours rapporté entre protagonistes.

Le tableau 4 montre la locutrice Stéphanie (droitière) qui incarne une vache (deuxième protagoniste du récit) en TP en train de dialoguer avec le cheval qui est tombé. Dans ces deux exemples produits à une seconde d'intervalle, la configuration de la main dominante est différente : sur l'image (1), elle figure le geste coverbal d'attendre tandis que sur l'image (2), elle figure le signe standard [ATTENDRE] en LSF. Cette seule différence ${ }^{6}$ suffit donc à faire varier la catégorie de transfert - il s'agit d'un TP en discours rapporté avec un emprunt à la gestualité humaine coverbale pour l'image (1), et d'un TP en discours rapporté en signe standard pour l'image (2) - et montre les possibles ressources utilisées par le locuteur pour faire dialoguer ses personnages transférés. Pour résumer, l'emploi de ces deux types d'unités (emprunt à la gestualité humaine d'une part et utilisation d'un signe standard d'autre part) suggère un choix différent de visée (illustrative d'une part, moins illustrative de l'autre).

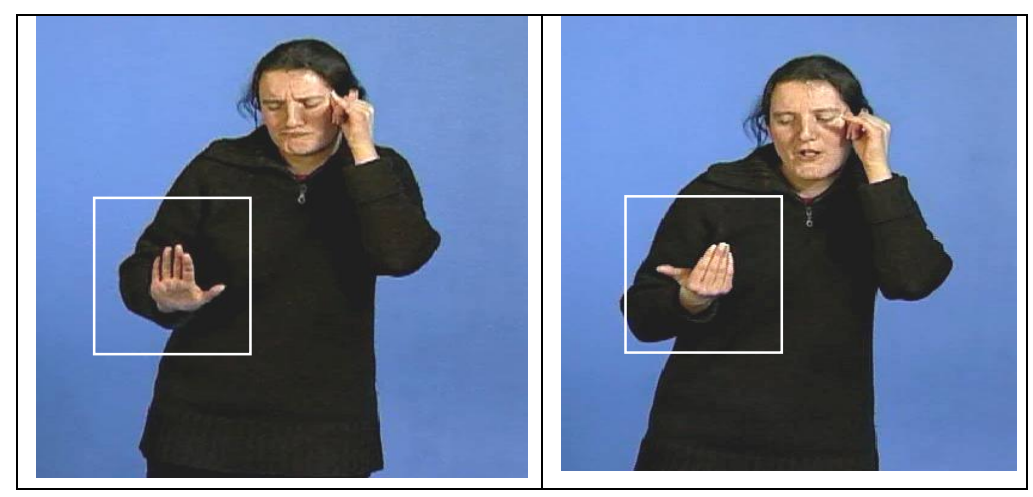

6 La mimique faciale est différente également, mais ceci n'entraine pas de changement notable puisque dans les deux cas le locuteur est en transfert personnel, donc la mimique faciale est celle du personnage transféré. 


\begin{tabular}{|l|l|}
\hline (1) $02 ' 12$ & (2) $02 ' 13$ \\
\hline $\begin{array}{l}\text { TP discours rapporté avec gestualité } \\
\text { signifiante }\end{array}$ & $\begin{array}{l}\text { TP discours rapporté avec signe } \\
\text { standard }\end{array}$ \\
\hline « attendre » & «ATTENDRE » \\
\hline
\end{tabular}

Tableau 4 : Exemple de deux unités en discours rapporté dans le récit du Cheval par Stéphanie

\subsection{Variation entre LS : l'exemple du signe standard [CHEVAL]}

Pour un référent animé simple (un cheval), il existe des variations lexicales non seulement au sein d'une même LS mais aussi entre LS variées. Au-delà de ces différences, l'intérêt est également de voir le «terreau » commun à ces signes qui se sont standardisés au fil du temps, à partir d'un même processus de l'iconicisation de l'expérience (voir infra). On souhaite voir également quelles SGI sont à l'origine de la création de ces signes, si on considère l'évolution diachronique des signes.

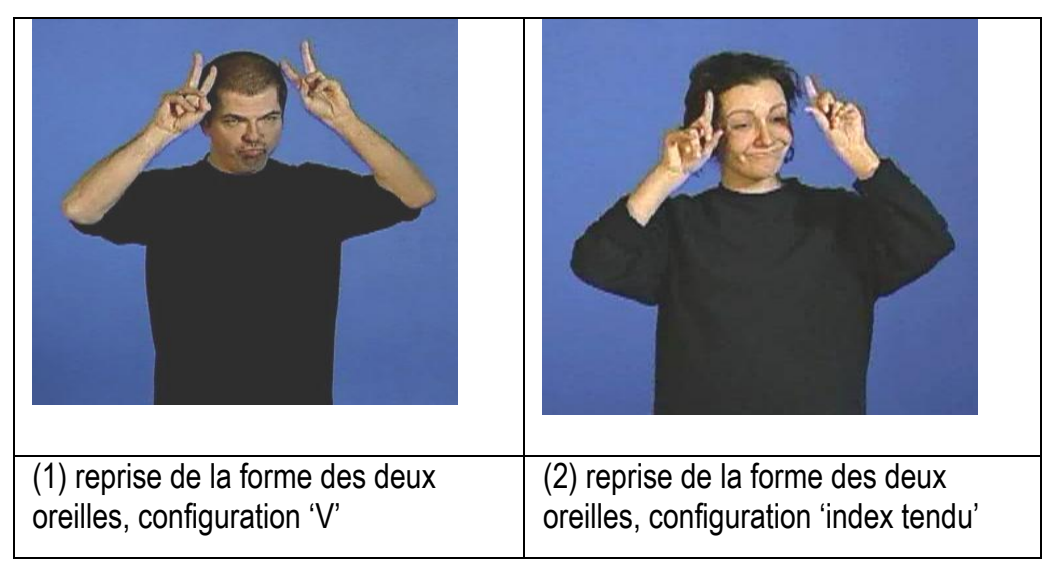

Tableau 5 : Deux variantes du signe standard [CHEVAL] en LSF

Dans le tableau 5, la SGI à l'origine de ces signes standard en LSF est un TTF, c'est la reprise manuelle de la forme d'une partie du référent, ici, les oreilles.

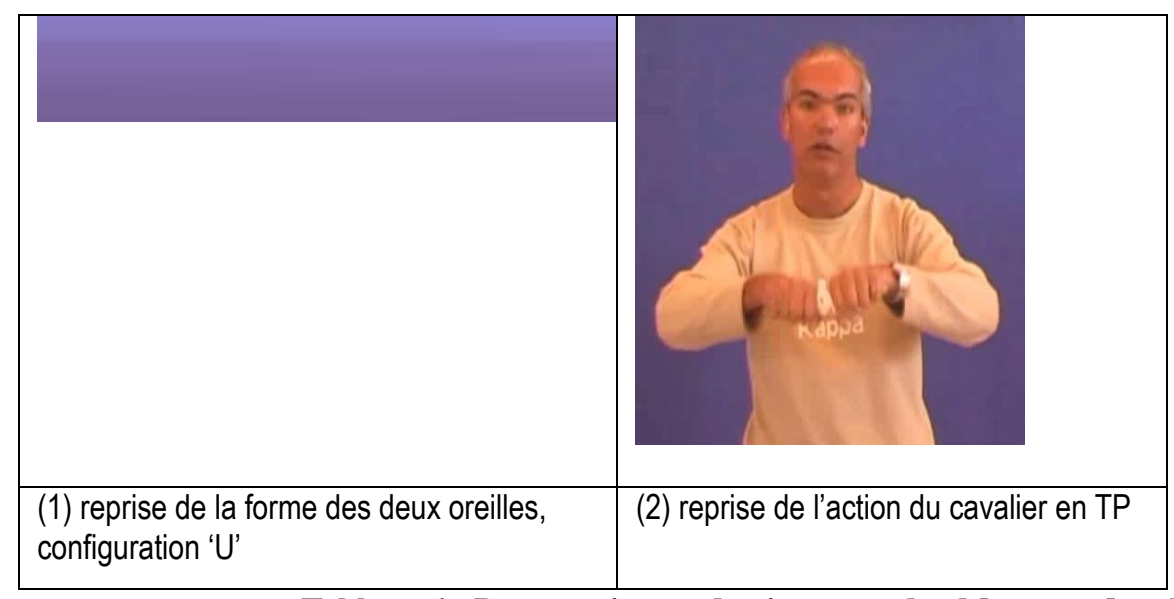

Tableau 6 : Deux variantes du signe standard [CHEVAL] en LS Mauricienne

Le tableau 6 présente deux variantes du signe [CHEVAL] en langue des signes mauricienne $(\mathrm{LSM})^{7}$. Les SGI à l'origine de ces signes standard sont un TTF pour (1) et un TP pour (2). Le signe

7 Cette langue est encore a un stade récent de son institutionnalisation, puisqu'une école pour enfants sourds existe depuis seulement quarante ans à l'île Maurice et que les recherches linguistiques sur cette langue ont réellement commencé en 2004, avec notamment la parution d'un premier dictionnaire de LSM (Gebert et Adone, 2006) et le développement d'activités de promotion de cette langue. 
(1) est très proche du signe en LSF, ce qui suggère une possible influence de la LSF sur la LSM.

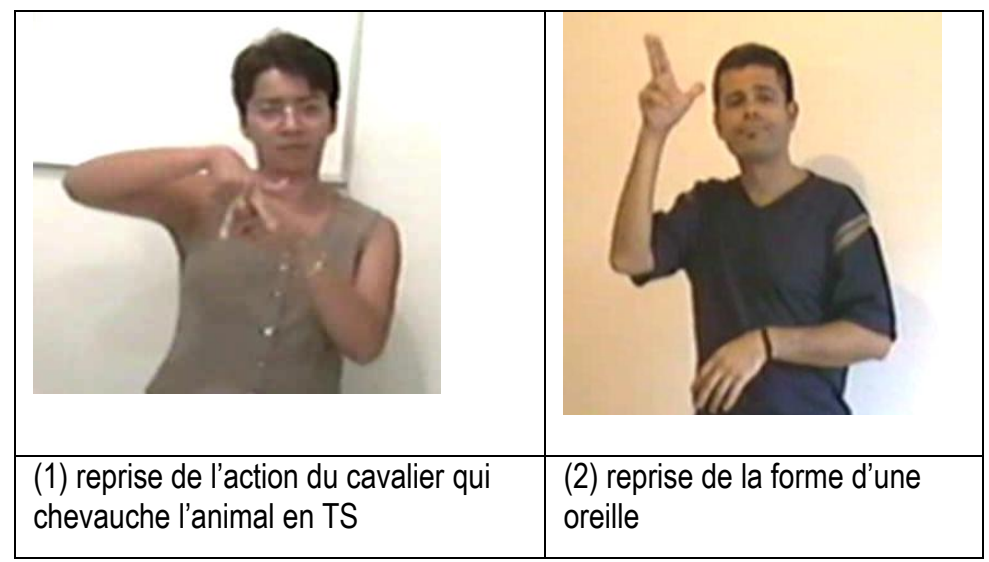

Tableau 7 : Deux variantes du signe standard [CHEVAL] en LIBRAS

Enfin, dans ces exemples en LIBRAS (langue des signes brésilienne) d tableau 7, les SGI à l'origine de ces signes standard sont un TS pour (1) et un TTF pour (2).

Certaines différences ont donc été observées dans les LS étudiées, en fonction de l'iconon (Bonnal, 2005, et voir l'introduction générale) exprimé. Au final, trois grandes stratégies ont été observées dans la signation du signe [CHEVAL], quelque soit la LS : a) reprise d'une partie de la forme du référent (les oreilles, par une ou deux mains, et avec différentes configurations manuelles); $b$ ) reprise de l'action du cavalier en transfert personnel; c) enfin, reprise de l'action du cavalier qui chevauche l'animal en transfert situationnel.

Mais ces exemples de signes lexicalisés font également ressortir de fortes similitudes lexicales entre LS. Celles-ci concernent surtout les paramètres non manuels, à savoir : labialisation nette du signe (le plus souvent), regard net vers l'interlocuteur, position du buste face à l'interlocuteur, et signe effectué plus rapidement qu'une SGI.

Pour résumer les différences entre pantomime et SGI, on peut dire que d'un côté, dans la pantomime, on a le maintien d'une globalité pour exprimer sur le corps les effets du monde et, de l'autre, pour les SGI, on a le maintien d'un morcellement pour exprimer le monde par les effets du corps. D'un côté la pantomime dont les effets du monde soumettent le corps à des durées variables dépendantes des actions mimées, laissent une gamme d'une telle étendue de tempi qu'elle ne favorise par l'émergence d'unités stabilisantes, c'est-à-dire d'un lexique. De l'autre, les SGI pour lesquelles le corps exprimant le monde par ses propres effets, permettent d'exploiter ces effets jusqu'à l'affranchissement de l'apesanteur et de l'inertie. Ceci favorise l'utilisation d'un tempo interne qui homogénéise la stabilisation temporelle des unités, d'où l'émergence possible des unités lexicales. 


\section{Sémiogenèse des Langues des Signes : de l'iconicisation de l'expérience à l'émergence et à la stabilisation des formes linguistiques}

Complétant le cheminement à travers le continuum, et ce dans le cas d'analyse des systèmes de communications gestuels mis en place par des individus sourds avec leur entourage entendant, nous allons voir $1 /$ comment, dans ce cadre, l'iconicisation de l'expérience au moyen des SGI diffère aussi de la pantomime ; 2 / comment la variation et la stabilisation de formes sont attestées, et 3/ comment l'incidence de la gestualité des entendants sur ces types de LS amène à reconsidérer l'écart entre gestuelle et LS. Ces systèmes, étudiés dans le cadre du modèle sémiogénétique (voir intro BG) sont dénommés langues des signes émergentes ${ }^{8}$ (Fusellier-Souza, 2004) et nous intéressent tout particulièrement puisqu'ils donnent une vision en synchronie de ce qui ont été les premiers stades d'évolution des LS. Des études sur les LS émergentes (Kendon, 1980 ; Yau, 1992 ; Morford, 1996 ; Goldin-Meadow, 2003 ; Fusellier-Souza, 2001, 2004, 2006), ont démontré que les individus sourds vivant exclusivement en environnement entendant ont mis en œuvre des stratégies communicationnelles communes fondées sur l'iconicisation de l'expérience. L'analyse du fonctionnement de ce processus permet de mettre en évidence certains procédés linguistiques qui sont à l'origine des conditions d'émergence des LS à la fois similaires (individu sourd en interaction gestuelle avec un entendant) et à la fois spécifiques à l'environnement familial et socioculturel.

\subsection{Incidence de la gestualité naturelle dans l'évolution et structuration des LS émergentes}

Les études portant sur les LS émergentes des adultes sourds pratiquées en environnement entendant on montré que l'input visuel et gestuel auquel l'individu sourd est exposé a des incidences sur la formation de son système linguistique. Toutefois, l'impact de cette influence extérieure dépendra de l'environnement et de la culture dans laquelle vit la personne sourde. En effet, l'utilisation de la gestualité naturelle par les entendants est liée à différents facteurs socioculturels. Dans des pays où la population utilise massivement les gestes accompagnant la parole dans la communication quotidienne, la source d'entrée gestuelles dans les LS émergentes sera plus productive. C'est ce que montrent les études de Sorin-Barreteau (1996) sur le langage gestuel de Mofu-Gudur au Nord-est du Cameroun et Jirou (2001) sur une LS informelle pratiquée à Mbour au Sénégal. Jirou (2008 : 21) signale que « l'enfant sourd, né dans une communauté d'entendants [en Afrique], ne subit pas l'isolement (ou dans une moindre mesure), que peuvent éprouver les sourds de France ou d'autres pays ayant perdu leur traditions orales. Il acquiert tout jeune le lexique signé des entendants et cet environnement gestuel, participant à sa communication naturelle, est propice à la création d'autres signes pour enrichir son système linguistique ». L'étude de Fusellier (2004) portant sur les LS

8 Ces systèmes linguistiques sont aussi désignés dans la littérature par les termes homesigns, idiolectes gestuels ou encore langues des signes spontanées. 
émergentes des adultes sourds brésiliens suggère également l'existence d'une influence avérée de la gestualité de l'environnement entendant dans la formation du stock lexical des LS émergentes.

\subsection{Les structures linguistiques des LS émergentes de sourds adultes brésiliens}

Fusellier (2004) décrit en détail le fonctionnement linguistique de trois LS émergentes pratiquées par des sourds adultes au Brésil. L'analyse s'appuie sur des corpus vidéo d'interactions entre locuteurs sourds (Jo, Ivaldo et Ana) avec leurs interlocuteurs entendants privilégiés (Manuel frère de Jo, Lucia épouse d'Ivaldo et Joana, sœur d'Ana). Ces personnes sourdes n'ont pas de contact entre elles ni avec d'autres Sourds mais bénéficient d'une certaine intégration sociale : Jo travaille avec l'un de ses frères dans la préparation de pizzas, Ivaldo est un vendeur ambulant de jus de fruits et Ana n'a pas d'activité professionnelle, mais elle participe aux activités domestiques et à la vie de son quartier. Les LS émergentes pratiquées par ces trois locuteurs sourds sont structurellement et fonctionnellement semblables et montrent des propriétés similaires avec d'autres LS émergentes (enfantines au adultes) étudiées dans la littérature : un stock lexical stabilisé (certains émanant de la gestualité environnante) à valeur nominale, verbale, adjectivale et des quantificateurs, des structures de grande iconicité et des pointages à fonctions multiples.

Un travail de transcription détaillée a permis d'extraire 3074 occurrences de différents types de signes gestuels produits pendant 15 minutes de discours de chaque locuteur sourd en interaction avec son interlocuteur. La catégorisation des unités gestuelles a été réalisée à partir d'une typologie des signes en quatre grandes catégories fonctionnelles: 1/ unités lexicales stabilisés, 2/unités gestuelles de Grande Iconicité (structures de transferts), 3/ pointages et 4/ unités gestuelles ayant trait à la gestualité conversationnelle brésilienne.

\subsection{Aperçu quantitatif des données}

L'analyse quantitative montre la corrélation entre les différentes catégories de signes par locuteurs sourds et entendants (interlocuteurs privilégiés). Les pourcentages respectifs de chaque catégorie sont illustrés par le graphique ci-dessous : 


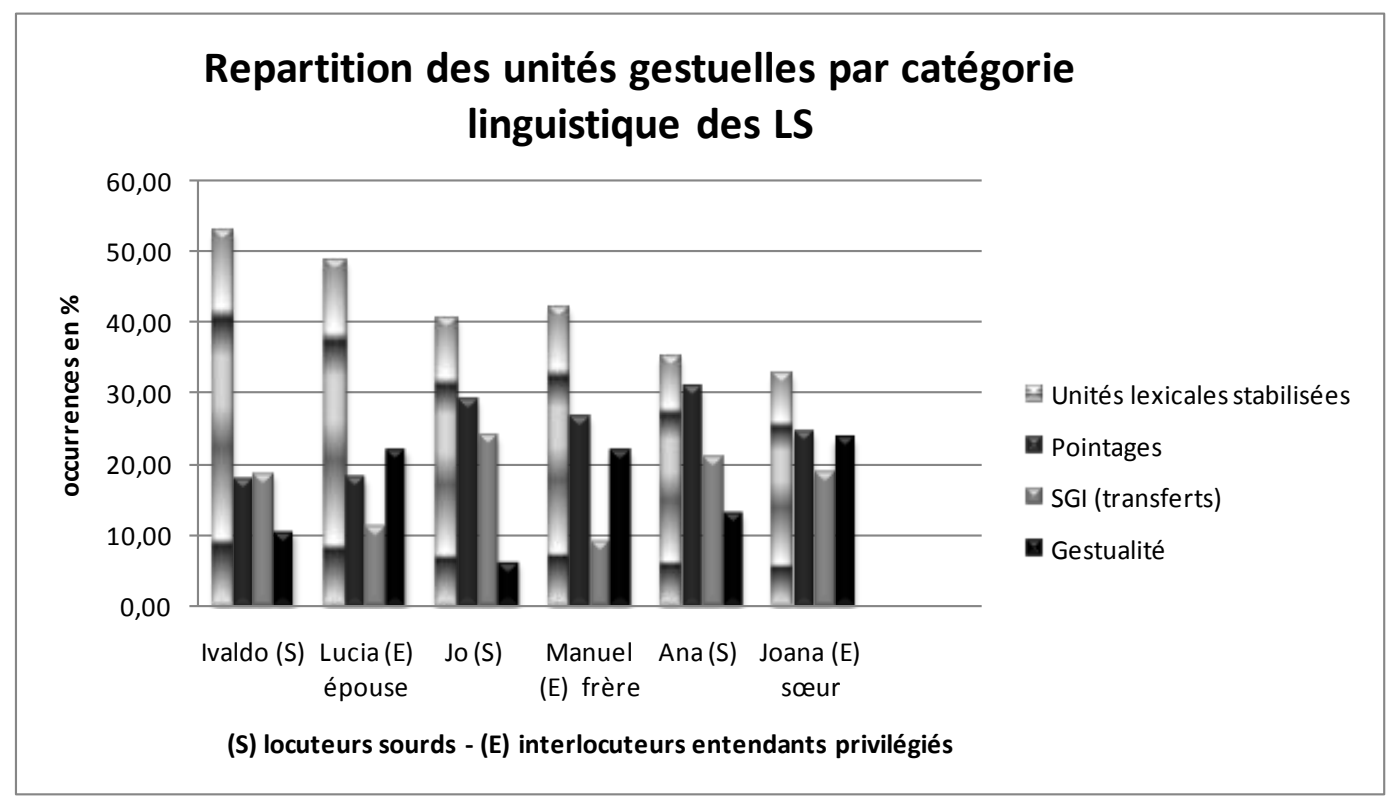

Graphique 1 : Répartition d'unités gestuelles par catégorie linguistique des LS

Ce graphique révèle une utilisation plutôt structurée et harmonieuse entre interactants des quatre catégories d'unités gestuelles dans les 3 LS émergentes. La présence d'unités lexicales stabilisés et des SGI permettent d'entrevoir que la complémentarité fonctionnelle entre les deux manières de dire en LS (dire en montrant et dire sans montrer) est déjà attestée dans ces LS. Les unités lexicales stabilisées, premiers indices de la variation et de la stabilisation de formes spécifiques à l'environnement familial et socio-culturel, se sont constituées d'une part, à partir de l'input de la gestualité environnante et d'autre part, à partir d'un processus de routinisation des unités de transferts (SGI) qui ont perdu leur visée illustrative, comme l'atteste le processus de développement de toutes les LS.

\subsection{Impact de la gestualité environnante sur les 3 LS émergentes}

L'analyse de corpus montre une utilisation authentiquement linguistique du canal visuo-gestuel lors des interactions. On remarque la présence :

- D’un « réseau d'influences mutuelles » (Kerbrat-Orecchioni, 1990) entre les participants du discours marqué essentiellement par des postures corporelles, par la direction du regard, des coups d'œil discontinus et des hochements de tête.

- De signes gestuels régulateurs de l'interaction : capteurs d'attention (signes gestuels d'appel) ; les énoncés des locuteurs sont ponctués de signes à valeur de questions : «tu vois ? », «tu comprends ? », «alors? ».

- De mouvements corporels (l'orientation du corps) marquant la distance proxémique entre les locuteurs. 
- De l'utilisation pertinente du regard dans la structuration de l'interaction : la direction du regard permet entre autres, d'indiquer le début, la suite et la fin de l'échange discursif.

Parallèlement aux aspects pragmatiques et interactionnels, des aspects fonctionnels et formels ont été dégagés dans la production gestuelle des interlocuteurs entendants. Ceux-ci sont capables :

- De mettre en forme gestuelles au moyen des structures de transferts un concept n'ayant pas d'unité gestuelle lexicale à partir de transferts de taille et de forme dans une visée illustrative et explicative. Ces constructions s'actualisent par l'activation du regard. Ceci est illustré dans l'exemple (1) :

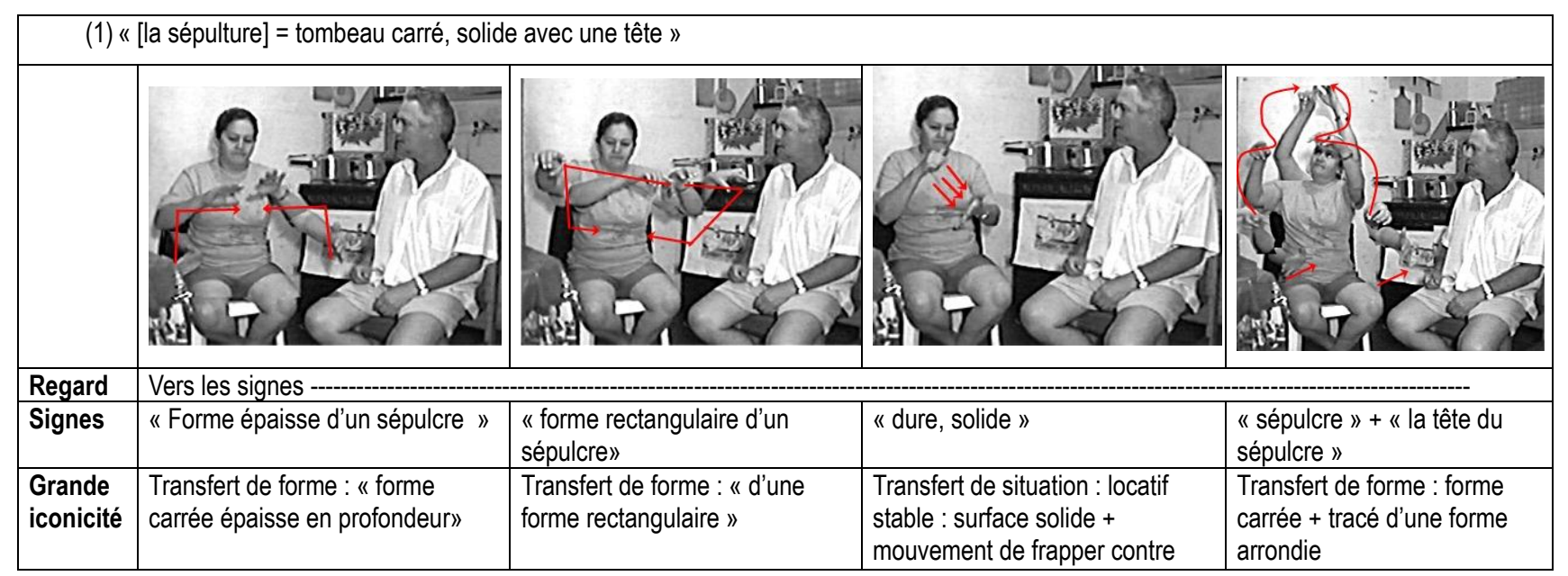

Tableau 8 : Exemple de construction du dire fondé par l'iconicisation de l'expérience au moyen des SGI

Dans le répertoire lexical des locuteurs sourds, certains signes sont issus de la gestualité brésilienne. Dans les exemples (2) et (3), Jo et Ivaldo ont le même signe pour désigner le concept de 'radin' (geste de faire le poing fermé en le retenant). En (4) et (5), Ivaldo utilise le signe «avoir des grands yeux » pour désigner le concept 'd'envieux'. Les deux signes semblent avoir été influencés par des métaphores existant également dans des expressions de la langue vocale du portugais du Brésil (ter olho grande) et de la gestuelle brésilienne (mão de vaca ou mão presa).

\begin{tabular}{|c|c|c|c|}
\hline (2) « radin » & $(3)$ « radin » & $(4)$ « envieux » & (5) « envieux » \\
\hline & &
\end{tabular}

Tableau 10 : Exemple des signes lexicaux stabilisés issus de la gestualité entendante

L'intégration sociale et la nature des échanges avec l'entourage sont déterminantes dans la qualité et la quantité du répertoire des signes de chaque LS émergente. Par exemple, Ivaldo et Jo, en raison de leur intégration dans le monde du travail, possèdent un grand nombre des signes pour représenter 
leurs fonctions dans leur univers professionnel. Ana, bien que son univers perceptivo-pratique soit restreint à son environnement familial, possède pourtant un riche répertoire gestuel pour les tâches ménagères, les activités sportives du quartier, la vie religieuse et la vie de famille. Par ailleurs, la création de certains signes lexicaux a été influencée par des comportements liés à la variable de sexe. Par exemple le signe stabilisé 'draguer' utilisé par Jo et Manuel en (6) et Ivaldo et Lucia en (7) se base sur le même ancrage perceptivo-pratique: «reprise d'une action prototypique de l'homme qui prend une femme dans ses bras » tandis que chez Ana et sa sœur, l'exemple (8) illustre que le signe s'inspire d'une autre représentation plus engagée et plus métaphorique : « reprise de deux bouches qui s'embrassent ».

(6) «draguer"

Tableau 11 : Exemple des signes lexicaux issus de l'ancrage perceptivo-pratique et des variables sociales

Ainsi, un remarquable processus de stabilisation lexicale a été observé dans le fil du discours. Dans l'exemple ( $9 \mathrm{a}$ et $9 \mathrm{~b})$, Jo fait référence au concept de «jeux vidéo », la première fois au moyen de structures de transferts; dans la deuxième mention, Jo utilise une forme plus économique morphologiquement, attestée par la suppression des formes périphériques et redondantes par le maintien d'une seule forme et par la réduction du temps de réalisation du signe.

\begin{tabular}{|l|c|}
\hline \multicolumn{1}{|c|}{$(9 \mathrm{a})$ « jeux vidéo » } & (9b) « jeux vidéo » \\
\hline Durée : 3 sec et 0,6 "de sec & Durée : 0,9 " de sec \\
\hline
\end{tabular}

Tableau 12 : exemple de processus de stabilisation lexicale à partir des SGI

Cet exemple met en évidence le principe d'économie linguistique et de stabilisation des formes dans le discours. Le processus de stabilisation lexicale se caractérise ainsi par au moins quatre indices : a) réduction des séquences illustratives, b) réduction de l'investissement corporel, c) simplification de la forme, d) désactivation du paramètre du regard dans la construction de la forme. Ce principe économique se caractérise par certaines primitives structurales qui sont similaires pour toutes les langues des signes : 
- Contrairement à ce que l'on peut constater avec la pantomime, le «dire » s'appuie sur une adéquation optimale entre forme perceptuelle et forme articulatoire contribuant à une mise en forme verbale de l'univers de l'imagerie et de l'expérience ;

- Les structures de transferts basés sur l'iconicisation de l'expérience donnent naissance à un nombre considérable de signes productifs basé sur l'activation du dire en montrant ;

- Les unités de transferts basés sur les SGI peuvent subir un processus de condensation et de stabilisation de la forme (routines de transferts) et donnent naissance aux signes lexicaux stabilisés ;

Ces résultats réaffirment l'hypothèse de la bifurcation de visées et, en aval, valident le postulat d'un lien structural, en synchronie, entre les LS. Le schéma (tableau 13) reprend les principes d'organisation initiale du dire en LS proposés dans le modèle sémiogénétique.

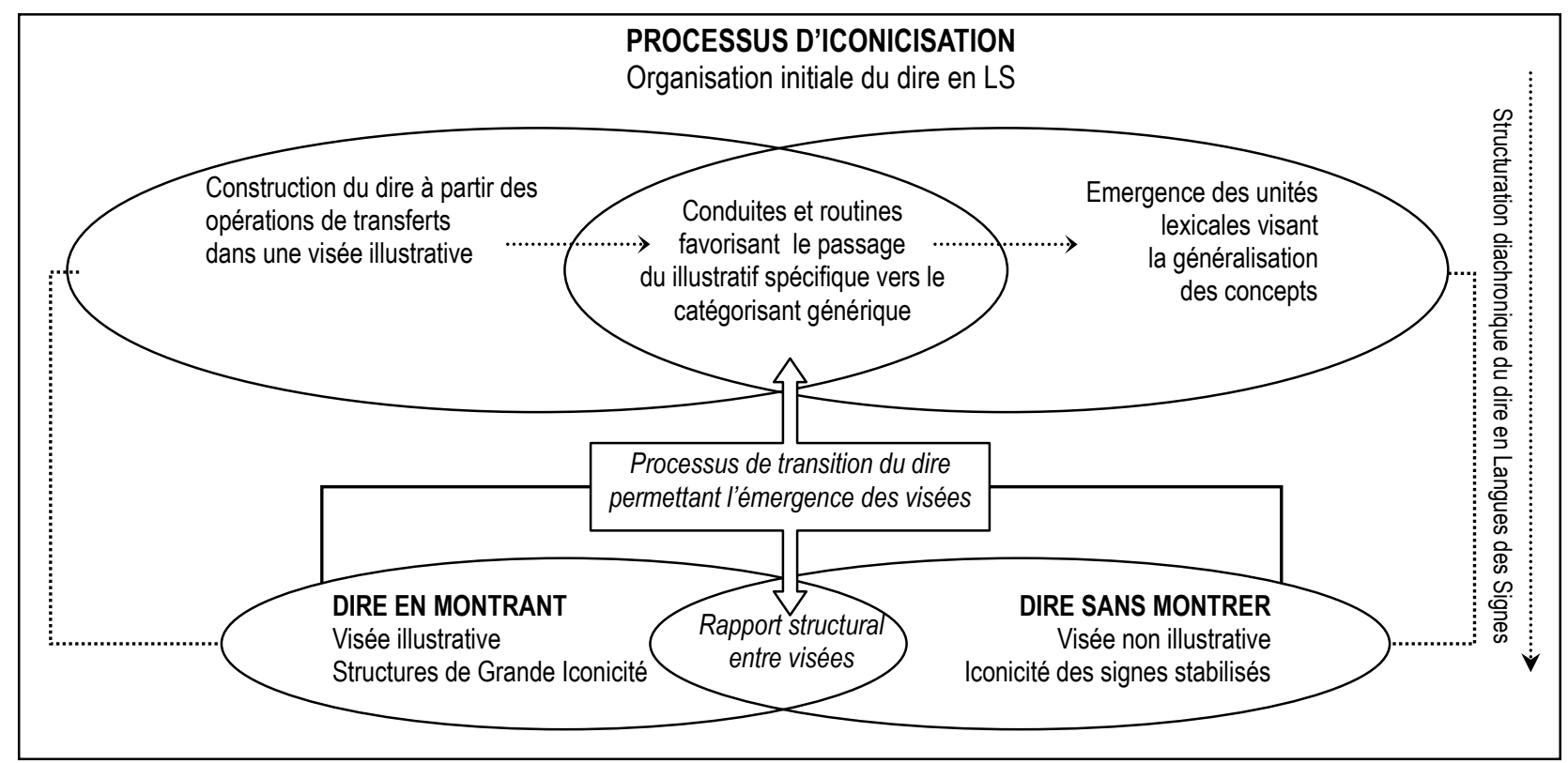

Tableau 13 : Etat des lieux du processus d'iconicisation et organisation initiale du dire en LS

L'analyse présentée ici a révélé que le processus initial d'iconicisation de l'expérience se construit selon une organisation élaborée obéissant à des principes structuraux propres aux LS. L'existence d'unités lexicales stabilisées et des SGI témoignent du fait que l'inscription du dire par ces locuteurs se fait déjà à partir d'une intentionnalité de visées (illustrative ou non). Ces données affichent l'intérêt de l'étude des LS émergentes pour comprendre le processus initial de constitution des LS et la nécessité de reconsidérer les questionnements sur la variation et le changement linguistique en LS et leur lien avec la gestualité entendante (intro BG). 


\section{Conclusion}

Cet écartement au plus large dans le continuum entre gestuelle d'un côté et LS de l'autre, connaît des exceptions. On vient de le voir, notamment dans la construction même de Langues des Signes émergentes. A cet égard, la multiplication des continua (4 au total) dont on a vu les biais qu'ils provoquent, résiste mal à ce principe de réalité qui voit se côtoyer les deux extrémités du continuum tant pour les LS émergentes que pour celles institutionnalisées comme la LSF. En effet, il suffit qu'un seul des axes de différenciation fasse défaut - celui de la parole - pour que se trouvent réunis et convoqués dans l'émergence de LS ce qu'il reste des instances du continuum à l'exception de la pantomime.

\section{Références bibliographiques}

AUGUSTIN (1997) Les confessions [Confessionum], Paris, Editions Sand.

AUGUSTIN (1997) La doctrine chrétienne [De doctrina christiana], Paris, Institut d'études augustiniennes. AUGUSTIN (2000) Oeuvres. II, La Cité de Dieu [De Civitas Dei], Paris, Gallimard.

BOUTET D. (2001) : Approche morpho-dynamique du sens dans la gestuelle conversationnelle. Thèse de doctorat, Université Paris 8, Saint-Denis

BOUTET, D. (2008) «Une morphologie de la gestualité : une structuration articulaire», Cahiers de Linguistique Analogique $\mathrm{n}^{\circ} 5$, Dijon : ABELL.

CONDILLAC E., [1746] (2002), Essai sur l'origine des connaissances humaines, Paris, Vrin

CUXAC C. (1996) : Fonctions et structures de l'iconicité des langues des signes. Thèse de Doctorat d'Etat, Paris, Université Paris V.

CUXAC C. (2000) : La Langue des Signes Française; les Voies de l'Iconicité, Faits de Langues n¹5-16, Paris: Ophrys

CUXAC, C. (2005) : Des signes et du sens. In : Hombert J.-M. (ed.) Aux origines des langues et du langage, 196-211. Paris : Fayard.

CUXAC, C. \& SALLANDRE, M-A. (2007). Iconicity and arbitrariness in French Sign Language: Highly Iconic Structures, degenerated iconicity and diagrammatic iconicity. In Pizzuto, E., P. Pietrandrea, R. Simone (eds.) : /Verbal and Signed Languages: Comparing Structures, Constructs and Methodologies/. Berlin : Mouton de Gruyter. 13-33.

DELLA PORTA, G., (1655) [1586] La physionomie humaine, traduit du latin : De humana physiognomia, Roven, Berthelin

DIDEROT, D. (2000) [1751], Lettre sur les aveugles : à l'usage de ceux qui voient ; Lettre sur les sourds et muets : à l'usage de ceux qui entendent et qui parlent, Paris, Flammarion.

DUBOS, J-B., (1993) [1719], Réflexions critiques sur la poésie et sur la peinture, Paris, Ecole nationale supérieure des beaux-arts.

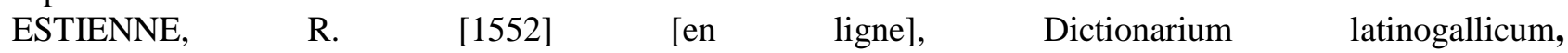
http://artfl.atilf.fr/dictionnaires/ESTIENNE/index.htm

FUSELLIER-SOUZA, I. (2001), La création gestuelle des individus sourds isolés. De l'Édification conceptuelle et linguistique à la sémiogènese des langues des signes. AILE 15. p. 61-96. Paris.

FUSELLIER-SOUZA, I. (2004), Sémiogenèse des langues des signes: Étude de langues des signes primaires (LSP) pratiquées par des sourds brésiliens. Thèse de doctorat, Université Paris 8, Saint-Denis.

FUSELLIER-SOUZA, I. (2006), Emergence and development of Signed Languages: from diachronic ontogenesis to diachronic phylogenesis. In Sign Language Studies, volume 7, $\mathrm{n}^{\circ}$ 1, Automne 2006. Gallaudet University Press. pp. 30-56.

GARCIA, B. (ce numéro) : Introduction du numéro. Présentation générale. Langage et Société ${ }^{\circ} \mathrm{xx}$.

GEBERT A. \& ADONE D. (2006) : Mauritian Sign Language (Langue des Signes Mauricienne - Lang Sign Morisien). Volume 1. Vacoas, Ile Maurice, Editions Le Printemps.

GOLDIN-MEADOW, S. (1991), « When does gesture become language? » A study of gesture used as a primary communication system by deaf children of hearing parents. In: Gibson, Kathleen Rita / Ingold, Tim (eds): Tools, language and cognition in human evolution. Cambridge : Cambridge Univ. Pr. pp. 63-85. 
GOLDIN-MEADOW S., 2003,. The Resilience of Language. Essays in developmental psychology, Psychology press, New York.

GRAF, F. (1991), Gestures and conventions: the gestures of roman actors and orators, in Jan Bremmer \& Herman Roodenburg (eds), A Cultural History of Gesture, Cambridge, Polity Press, pp 36-58

JIROU, G. (2001), « Description d'une langue informelle en dehors du milieu institutionnel. Analyse lexicale du parler gestuel de MBour (Sénégal) ». Article non publié.

JORIO de, A., KENDON, A. (2000) [1832], Gesture in Naples and Gesture in Classical Antiquity, Bloomington, Indiana University Press.

KENDON, A. (1980), «A Description of a Deaf-Mute Sign Language from the enga Province of Papua New guinea with Some Comparative Discussion ». Parte I : The formational properties of Enga signs. Parte II : The semiotic functioning of Enga signs; Parte III : Aspects of utterance construction In Semiotica 31, 1/2, 32, $1 / 2$ et $32,3 / 4$.

KENDON, A. (1988), How gestures can become like words. In Poyatos : Cross-Cultural Perspectives in Nonverbal Communication. Toronto, Hogrefe

KENDON, A. (2004), Gesture: Visible Action as Utterance, Cambridge, Cambridge University Press

KERBRAT-ORECCHIONI, C. (1990), Les interactions verbales. Paris. Armand Colin. Tome I, II.

LEBRUN, C., MOREL D'ARLEUX, L-M., (2000) [1806], De la physionomie humaine et animale, dessins de Charles Le Brun gravés pour la chalcographie du musée Napoléon en 1806, Paris, Edition de la réunion des musées nationaux.

LITTRE, E., (1956), [1863] Dictionnaire de la langue française, Paris, Jean-Jacques Pauvert Editeur.

MALLERY, G., (2001), [1881], Sign Language among North American Indians, Mineola, New York, Dover Publications.

McNEILL, D. (1992), Hand and Mind, What Gestures Reveal about Thought, Chicago, London, The University of Chicago Press.

McNEILL, D. (2000), Language and gesture, Cambridge, Cambridge University Press

Mc NEILL, D. (2005), Gesture and Thought, Chicago, London, The University of Chicago Press.

MORFORD, J.P. (1996), «Insights to language from the study of gesture: a review of research on the gestural communication of non-signing deaf people ». In Language \& Communication. Vol. 16, $\mathrm{n}^{\circ} 02$, Pergamon, London, pp. 165-178.

QUINTILIEN (1979), Institution oratoire. Tome VI, Livre X et XI, Paris, Les belles lettres

SALLANDRE, M.-A. (2003): Les unités du discours en Langue des Signes Française. Tentative de catégorisation dans le cadre d'une grammaire de l'iconicité. Thèse de doctorat, Université Paris 8 , SaintDenis.

SORIN-BARRETEAU L., (1996). Le langage gestuel des Mofu-Gudur, Thèse de doctorat, Université Paris $\mathrm{V}$ - René Descartes.

SCHMITT, J-C., (1990), La raison des gestes dans l'occident médiéval, Paris, Gallimard.

SINGLETON, J. L. \& Al (1995) : «The Cataclysmic Break Between Gesticulation and Sign: Evidence Against a Unified Continuum of Gestural Communication, in Karen Emmorey (ed), Language, Gesture, and Space, Hillsdale, Lawrence Erlbaum Associates Publishers, pp 287-311.

YAU, S. C. (1992), Création Gestuelle et début du Langage - Création de langues gestuelles chez les sourds isolés, éds Langages Croisés, Hong Kong. 